\title{
METRIC: A Dedicated Earth-Orbiting Spacecraft for Investigating Gravitational Physics and the Space Environment
}

\author{
Roberto Peron ${ }^{1, *}$ (D) and Enrico C. Lorenzini ${ }^{2}$ \\ 1 Istituto di Astrofisica e Planetologia Spaziali (IAPS-INAF), 00133 Roma, Italy \\ 2 Department of Industrial Engineering and CISAS “Giuseppe Colombo", University of Padova, \\ 35131 Padova, Italy; enrico.lorenzini@unipd.it \\ * Correspondence: roberto.peron@iaps.inaf.it; Tel.: +39-06-499-34-367 \\ Academic Editor: Darren L. Hitt \\ Received: 22 May 2017; Accepted: 13 July 2017; Published: 20 July 2017
}

\begin{abstract}
A dedicated mission in low Earth orbit is proposed to test predictions of gravitational interaction theories and to directly measure the atmospheric density in a relevant altitude range, as well as to provide a metrological platform able to tie different space geodesy techniques. The concept foresees a small spacecraft to be placed in a dawn-dusk eccentric orbit between 450 and $1200 \mathrm{~km}$ of altitude. The spacecraft will be tracked from the ground with high precision, and a three-axis accelerometer package on-board will measure the non-gravitational accelerations acting on its surface. Estimates of parameters related to fundamental physics and geophysics should be obtained by a precise orbit determination, while the accelerometer data will be instrumental in constraining the atmospheric density. Along with the mission scientific objectives, a conceptual configuration is described together with an analysis of the dynamical environment experienced by the spacecraft and the accelerometer.
\end{abstract}

Keywords: fundamental physics; general relativity; space environment; atmospheric density; International Terrestrial Reference Frame; measurement of acceleration; satellite laser ranging; global navigation satellite systems

PACS: 04.80.Cc; 06.20.f; 06.30.Gv; 07.87.+v; 14.70.Kv; 91.10.Fc; 91.10.Sp; 91.10.Ws; 95.10.Eg; 95.40.+s

\section{Introduction}

The near-Earth environment is an important place for performing experiments aimed at testing present models of the gravitational interaction. Gravitation is accurately described by the general theory of relativity by Albert Einstein, and no experiment carried out so far is in contradiction with the theory (see e.g., the recent detections of gravitational waves by the LIGO-VIRGO collaboration [1-4]). The overall validity of this picture is however being challenged by the theories that aim at reconciling general relativity with the microscopic domain (e.g., grand unification theories, quantum gravity), as well as by the mysteries of dark matter and dark energy, though mainly at scales from the galactic up to the cosmological. It is therefore important to precisely test the consequences of the theory, as well as those of competing ones at all the scales accessible with available experimental techniques. We may note that the range of scales up to now precisely tested extends to a few tens of AU [5]. In this respect, the range of scales corresponding to the semimajor axis of low Earth orbits (LEO) is a rather well-tested one [6,7], but room is left for improvements.

Among the various testing strategies, one of the most effective is the verification of the equation of motion of a gravitational test mass [8,9], as in the case (in many ways paradigmatic) of geodetic 
satellites [10]. This verification strategy requires the determination with a high degree of accuracy of the satellite trajectory (precise orbit determination-POD) and the subsequent use of this information for testing selected predictions of the theory.

The development of high-precision experimental space techniques, necessary for this type of tests, opens the way to complementary investigations. One of these deals with the accurate measurement of atmospheric drag in an altitude range where spacecraft operations, orbital evolution and lifetime estimations are significantly affected by this perturbation. Indeed, after more than fifty years of space activity, the knowledge of atmospheric density and its dependency on the solar and geomagnetic activity is still affected by relevant uncertainties [11,12]. Due to the expanding amount of man-made orbital debris [13], the improved knowledge of atmospheric density and its variability in LEO could also lead to a reduction of unneeded avoidance maneuvers.

In this paper, we expand on a proposed concept [14], which we name METRIC (Measurement of EnvironmenTal and Relativistic In-orbit preCessions), to make use of existing technology developed for acceleration measurement in space and state-of-the-art satellite tracking to precisely determine the orbit of a spacecraft with well-defined geometrical and mass characteristics and to measure over a long period of time the drag deceleration (as well as other non-gravitational perturbations) acting on it. This will result in a virtually drag-free spacecraft that can be exploited to investigate a number of effects related to gravitational physics and to the near-Earth environment, via a precise analysis of its orbital dynamics. Consequently, this mission would complement previous geodynamic and atmospheric ones in providing data useful to constrain competitive models of the gravitational interaction, as well as those related to atmospheric drag. In addition to this objectives, it turns out that such a spacecraft would naturally form the potential basis of a metrological platform useful to tie different space geodesy techniques.

After having set the main mission objectives and the strategies to achieve them, a model for mission configuration is introduced. A discussion of the dynamical environment experienced by the spacecraft is a prerequisite for a quantitative assessment of non-gravitational accelerations that will be sensed by the on-board accelerometer.

\section{Mission Objectives}

We identified the following broad-range objectives for METRIC, in a $450 \times 1200 \mathrm{~km}$ high-inclination orbit:

1. conduct fundamental physics tests by verifying the general relativistic equation of motion of a test mass, placing limits on alternative theories of gravitation;

2. improve the knowledge of selected tidal terms;

3. estimate, through acceleration measurements, the atmospheric density in the orbital region of interest;

4. establish a space-based tie among different space geodesy techniques.

In the first two objectives, the basic feature of having a de facto gravitational test mass is employed. This is an idealized concept (see, e.g., [9]): the accuracy of any materialization of it depends on how much the non-gravitational perturbations are accounted for and on how well the spacecraft metrology is implemented.

The IERS (International Earth Rotation and Reference Systems Service) Conventions (2010) [15] recommend that the following relativistic correction to the accelerations of a test mass in the Geocentric 
Celestial Reference System (GCRS) be used (here and afterwards, the dot denotes derivative with respect to time):

$$
\begin{aligned}
\Delta \ddot{\mathbf{r}}= & \frac{G M_{\oplus}}{c^{2} r^{3}}\left\{\left[2(\beta+\gamma) \frac{G M_{\oplus}}{r}-\gamma \dot{\mathbf{r}} \cdot \dot{\mathbf{r}}\right] \mathbf{r}+2(1+\gamma)(\mathbf{r} \cdot \dot{\mathbf{r}}) \dot{\mathbf{r}}\right\}+ \\
& (1+\gamma) \frac{G M_{\oplus}}{c^{2} r^{3}}\left[\frac{3}{r^{2}}(\mathbf{r} \times \dot{\mathbf{r}})\left(\mathbf{r} \cdot \mathbf{J}_{\oplus}\right)+\left(\dot{\mathbf{r}} \times \mathbf{J}_{\oplus}\right)\right]+ \\
& \left\{(1+2 \gamma)\left[\dot{\mathbf{R}} \times\left(\frac{-G M_{\odot} \mathbf{R}}{c^{2} R^{3}}\right)\right] \times \dot{\mathbf{r}}\right\}
\end{aligned}
$$

In this expression, $c$ is the speed of light; $\beta$ and $\gamma$ are PPN (parameterized post-Newtonian) parameters; $\mathbf{r}$ is the test mass position with respect to the Earth; $\mathbf{R}$ the position of the Earth with respect to the Sun; $\mathbf{J}_{\oplus}$ is the Earth's angular momentum per unit mass; and $G M_{\oplus}, G M_{\odot}$ are the gravitational coefficients of the Earth and Sun, respectively. The three terms on the right-hand side of Equation (1) can be identified with the so-called gravitoelectric, gravitomagnetic and geodetic (de Sitter) contributions, respectively. This formulation derives from the PPN framework and is considered to be adequate in the weak-field, slow-motion conditions of the Solar System [16]. It can be predicted (via first-order perturbation theory; see, e.g., $[8,17]$ ) that, due to those terms, the orbit argument of pericenter and longitude of ascending node are subjected to secular precessional effects (see Section 4.2).

The equation of motion, along with its predictions, can be tested against the orbit as obtained by the POD. Of course the dynamics underlying this orbit is much more complex than the model described by Equation (1): in short, an Earth-bound spacecraft is subjected to the non-spherically symmetric and time-dependent Earth gravitational field and to an ensemble of small, but non-negligible non-gravitational forces. On one hand, this implies the possibility of improving the knowledge of geophysical features to which the selected orbit is more sensitive; on the other hand, one has to constantly face the issue of the non-gravitational perturbations, which in this context constitute a noise that potentially masks the gravitational signal to be measured. Regarding this second issue, the role of the accelerometer is exactly that of enabling a disentangling of the two parts.

We notice that the proposed semimajor axis and eccentricity make the orbit more sensitive to Earth tides (both solid and ocean) with respect to higher altitude geodetic satellites, like the two LAGEOS. In this respect, the dynamical environment that METRIC will encounter is much more similar to the one faced by Starlette [18]. This implies a remarkable sensitivity to a spectrum of solid and ocean tides and in turn points to interesting possibilities for improving our knowledge of tides and, more in general, mid- to high-wavelength features of the Earth's gravity field.

The third objective foresees a different use of the acceleration observables. While for the previous two objectives, as said, one wants to measure a noise that could bias the parameters' estimate, in this case, one measures a signal that is related to the local atmospheric drag to be measured. A $450 \times 1200 \mathrm{~km}$ high-inclination orbit would enable the sampling of an important circumterrestrial region to improve the knowledge of neutral atmospheric density that would be relevant for both space operations and science. The altitude range considered is characterized by a compositional and density landscape strongly affected by the time-varying solar activity. Unlike previous estimates of atmospheric density (obtained from satellite orbital decay and therefore being averages over many orbits), the on-board accelerometer will allow instantaneous measurements over the altitude range of interest.

The fourth objective is related to the spacecraft tracking and system configuration, discussed in Sections 3 and 6, respectively. Indeed, the multi-technique approach that is proposed could in principle enable a space-based tie among the space geodesy techniques involved in each of the chosen tracking options. This idea is the basis for the Geodetic Reference Antenna in Space (GRASP) proposal [19]. Such a space-based tie would help to improve the coherence of the geodetic products based on these techniques, e.g., the International Terrestrial Reference Frame (ITRF) [20]. 
The basic idea is to have a well-known metrological platform that could host different space geodesy techniques, realizing the space equivalent of what in geodesy is known as a local tie. This platform would be calibrated accurately on the ground and would provide a stable reference to link the various realizations of the Terrestrial Reference Frame, also helping to calibrate, e.g., specific space geodetic devices.

A satellite like METRIC could be considered to be somewhere in the middle between LAGEOS and CHAMP [21-23]. The former (along with its twin LAGEOS II and the recently-launched LARES) is a good example of an object developed, built and launched to work as a gravitational test mass. It is completely passive (no instrumentation or any devices on-board); therefore, any dynamical perturbing effect acting on it should be a posteriori modeled, either analytically or numerically. In CHAMP, the effects of non-gravitational origin are directly measured by a three-axis accelerometer, made necessary by the need to remove the combined contributions of non-gravitational perturbations from the satellite-to-satellite tracking data, of which the air-drag is the dominant effect at the orbital altitude of CHAMP (this is also the choice done in the BepiColombo mission concept, again due to the complexity of the non-gravitational effects acting on the spacecraft [24-26]). The proposed mission retains the basic idea of having an object of a relatively simple shape, including a three-axis accelerometer package in order to overcome the problems related to modeling the non-gravitational effects and, at the same time, separating the air-drag from the solar-pressure effects, as explained later on. In this respect, METRIC can be seen as an evolution of CHAMP (with a focus on gravitational physics, geodesy and atmospheric physics), keeping the spacecraft segment as simple as possible, compatibly with the mission requirements.

To summarize, the core element of METRIC is the availability of a small platform that can act at the same time as a gravitational test mass (whose orbit is in principle only sensitive to its gravitational dynamics), as a drag-sensing device (able to measure the instantaneous and not only integrated atmospheric density) and as a space-based target for geodetic measurements (providing a tie among different space geodesy techniques). The main elements to be defined and implemented in order to fulfill these broad-range requirements will be discussed in the following.

\section{Mission Configuration}

In order to satisfy the scientific objectives, one needs an orbiting object with very well-characterized physical properties, hosting a three-component accelerometer package and all of the required ancillary instrumentation and subsystems. State-of-the-art tracking of the satellite is also assumed. The orbit will be elliptical, spanning an altitude range from 450 to $1200 \mathrm{~km}$, where drag-related effects impact significantly the lifetimes of satellites. The spacecraft should be cylindrical in shape and spinning about its longitudinal z-axis, which will be close to orthogonal to the orbital plane. The three-axis accelerometer package will need an acceleration resolution better than $1 \times 10^{-10} \mathrm{~m} \cdot \mathrm{s}^{-2}$. The expected measurement range is $1 \times 10^{-7}$ to $1 \times 10^{-10} \mathrm{~m} \cdot \mathrm{s}^{-2}$ considering estimates of drag forces at minimum and maximum solar activity conditions in the altitude range of interest and a preliminary estimate of the satellite $A / m$ ratio (see the discussion in Section 4 and, in particular, the estimates provided in Table 1).

The preliminary choice for our analysis is a dawn-dusk, Sun-synchronous orbit. This choice of orbit favors the evaluation of the drag-related accelerations and resolving them from the solar pressure-related accelerations. In the altitude range of METRIC, the solar radiation-related acceleration is of the same order of magnitude of the air drag-related acceleration at perigee altitudes, but it is about two orders of magnitude stronger than air drag at the apogee altitudes. The separation of the two effects and the possibility of extracting the much weaker air drag acceleration from the acceleration data are attained through frequency modulation (i.e., at the spin frequency of the spacecraft) and the orbital/geometrical configuration adopted. The dawn-dusk, Sun-synchronous orbit also provides an almost constant illumination (useful for electric power generation and thermal stability) and very short periods of eclipses close to the winter solstices. Different choices of orbital inclinations (e.g., polar) 
that would favor the analysis of the relativistic effects, but lose the features mentioned above will be analyzed in follow-up studies.

Table 1. Expected amplitudes of accelerations acting on METRIC for a $450 \times 1200 \mathrm{~km}$ Sun-synchronous orbit, with an altitude range of 471 to $1221 \mathrm{~km}$ above the reference ellipsoid.

\begin{tabular}{|c|c|c|}
\hline Acceleration Type & Range $\left(\mathbf{m} \cdot \mathbf{s}^{-2}\right)$ & Remarks \\
\hline $\begin{array}{c}\text { Neutral drag: } \\
\text { LH } \\
\text { LV } \\
\text { Side }\end{array}$ & $\begin{array}{l}3.4 \times 10^{-10} \text { to } 3.3 \times 10^{-7} \\
0 \text { to } 3.2 \times 10^{-9} \\
8.0 \times 10^{-12} \text { to } 1.4 \times 10^{-8}\end{array}$ & $\begin{array}{l}\text { LH component of neutral drag is the } \\
\text { "signal" to be extracted }\end{array}$ \\
\hline $\begin{array}{c}\text { Solar radiation pressure: } \\
\text { transverse axes } \\
\text { Side }\end{array}$ & $\begin{array}{c}1.2 \times 10^{-9} \text { to } 3 \times 10^{-9} \\
\sim 5 \times 10^{-8}\end{array}$ & $\begin{array}{l}\text { Assume the satellite body shielded from } \\
\text { direct solar rays by solar panel }\end{array}$ \\
\hline $\begin{array}{l}\text { Satellite spin motion: } \\
\text { transverse axes } \\
\text { Side }\end{array}$ & $\begin{array}{c}\sim 2.7 \times 10^{-6} \\
0\end{array}$ & $\begin{array}{l}\text { Assume a coning (nutation) angle } \\
\text { of } 1 \times 10^{-4} \mathrm{rad}\end{array}$ \\
\hline $\begin{array}{l}\text { Gravity gradients: } \\
\text { transverse axes } \\
\text { Side }\end{array}$ & $\begin{array}{l}3.3 \times 10^{-9} \text { to } 4.5 \times 10^{-9} \\
1.3 \times 10^{-9} \text { to } 9.2 \times 10^{-10}\end{array}$ & $\begin{array}{l}\text { Intensities are for sensing mass located } \\
\text { farthest away from the satellite CM: } 5 \mathrm{~cm} \\
\text { along the } \mathrm{z} \text { axis and } 1 \mathrm{~mm} \text { radially }\end{array}$ \\
\hline
\end{tabular}

The spacecraft cylindrical body should be in the shadow of a flat, round-shaped solar panel facing the Sun (see Figure 1). This arrangement will keep the effects of the solar radiation pressure at a right angle, for both the direct illumination on the solar panel and the albedo and infrared radiation from Earth, with respect to the main contribution of the drag force along the flight direction. The spin about an axis close to orthogonal to the orbit plane provides the basic attitude stabilization of the spacecraft to be sporadically adjusted by the attitude control system. This arrangement keeps the spacecraft facing the flight direction with a constant cross-sectional area while the spin modulates the components in the orbital plane (in-plane) of the surface forces, inclusive the LH component (see later) of the drag-related acceleration.

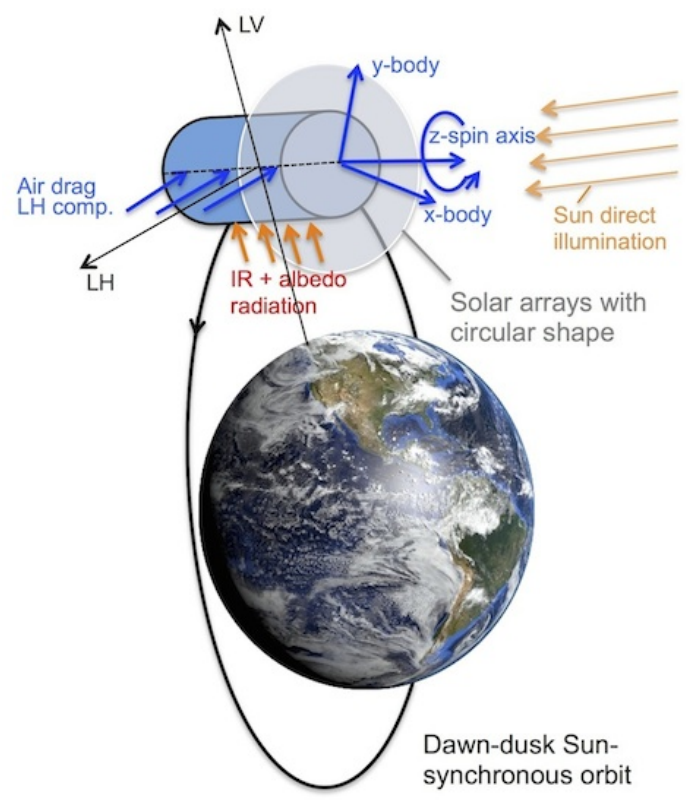

Figure 1. Schematic of the proposed METRIC spacecraft and orbit. This configuration enables a separation among different dynamical effects. 
The proposed orbit is characterized by a rather high eccentricity $e=0.052$ that provides the observable (i.e., the argument of the pericenter), sensitive to a series of effects: relativistic contributions (see, e.g., [27-30]) and Earth's tides. The nodal longitude will be also an important observable for fundamental physics tests.

With this orbit, estimated values for various effects regarding the orbit evolution in time can be obtained; see the discussion in Section 4 and the values provided in Table 2. As expected, the Schwarzschild precession of the argument of pericenter is the dominant relativistic signal that should emerge from the noise due to a rather low orbit and a well-identifiable pericenter (in the case of a quasi-circular orbit, which is the case of many geodetic satellites, the argument of pericenter is a quantity properly defined, but in practice not easily detectable; this is, e.g., the case of LARES, whose orbit is characterized by an eccentricity of $\sim 1 \times 10^{-3}$ ). The gravitomagnetic contributions have a smaller signal-to-noise ratio. The advantage of using high-inclination orbits (e.g., Sun-synchronous or polar) is a significant suppression of the competitive precession signal on the node associated with the uncertainty of the knowledge of the Earth's lower zonal gravitational harmonics (in particular, the quadrupole). The rough estimates presented here point to potentially interesting possibilities for a single-satellite measurement of gravitational effects (possibilities that in any case should be confirmed by an error analysis more detailed with respect to the one presented here). Moreover, the data could be combined with those originated from other geodetic missions (this is an interesting possibility, which has been proposed and discussed many times in the past, and found an effective application, e.g., in the relativistic tests performed by combining the Keplerian elements' residuals of the three geodetic satellites LAGEOS, LAGEOS II and LARES; adding other satellites to the list could increase the probability of finding even more suitable observables than the ones employed so far), thereby broadening the range of possible measurements [31]. Potential improvements with respect to the state of the art $[10,29,30,32]$ include:

1. a measurement of pericenter precession improved by possibly one order of magnitude, with a corresponding constraint on a Yukawa-like contribution acting at a scale comparable with the orbit semimajor axis;

2. a direct (i.e., with a single satellite) measurement of Lense-Thirring precession.

Table 2. Estimates of relativistic (upper part) and Newtonian (lower part) precession rates for METRIC and relevant geodetic satellites; all values are in $\operatorname{arcsec} \mathrm{y}^{-1}$; arcsec stands for a second of arc. For each satellite, the orbital eccentricity has been explicitly indicated.

\begin{tabular}{ccccc}
\hline & METRIC & LAGEOS & LAGEOS II & LARES \\
\hline$e$ & $5.20 \times 10^{-2}$ & $4.43 \times 10^{-3}$ & $1.38 \times 10^{-2}$ & $1.20 \times 10^{-3}$ \\
\hline$\dot{\omega}_{\mathrm{Sch}}$ & 12.5 & 3.28 & 3.35 & 10.1 \\
$\dot{\Omega}_{\mathrm{LT}}$ & $1.53 \times 10^{-1}$ & $3.09 \times 10^{-2}$ & $3.17 \times 10^{-2}$ & $1.19 \times 10^{-1}$ \\
$\dot{\omega}_{\mathrm{LT}}$ & $6.93 \times 10^{-2}$ & $3.14 \times 10^{-2}$ & $-5.77 \times 10^{-2}$ & $-1.25 \times 10^{-1}$ \\
$\dot{\Omega}_{\mathrm{dS}}$ & $1.76 \times 10^{-2}$ & $1.76 \times 10^{-2}$ & $1.76 \times 10^{-2}$ & $1.76 \times 10^{-2}$ \\
$\dot{\omega}_{\mathrm{Y}}{ }^{*}$ & $1.44 \times 10^{-1}$ & $8.19 \times 10^{-2}$ & $8.29 \times 10^{-2}$ & $1.36 \times 10^{-1}$ \\
\hline$\dot{\Omega}_{\mathrm{N} 20}$ & $1.30 \times 10^{6}$ & $4.51 \times 10^{5}$ & $-8.31 \times 10^{5}$ & $-2.25 \times 10^{6}$ \\
$\delta \dot{\Omega}_{\mathrm{N} 20}$ & $1.42 \times 10^{-1}$ & $4.94 \times 10^{-2}$ & $9.10 \times 10^{-2}$ & $2.46 \times 10^{-1}$ \\
$\dot{\Omega}_{\mathrm{N} 40}$ & $-2.73 \times 10^{3}$ & $-2.50 \times 10^{2}$ & $9.07 \times 10^{1}$ & $2.99 \times 10^{3}$ \\
$\delta \dot{\Omega}_{\mathrm{N} 40}$ & $1.98 \times 10^{-2}$ & $1.81 \times 10^{-3}$ & $6.59 \times 10^{-4}$ & $2.17 \times 10^{-2}$ \\
$\dot{\omega}_{\mathrm{N} 20}$ & $3.91 \times 10^{5}$ & $3.06 \times 10^{5}$ & $1.01 \times 10^{6}$ & $1.57 \times 10^{6}$ \\
$\delta \dot{\omega}_{\mathrm{N} 20}$ & $4.28 \times 10^{-2}$ & $3.35 \times 10^{-2}$ & $1.10 \times 10^{-1}$ & $1.72 \times 10^{-1}$ \\
$\dot{\omega}_{\mathrm{N} 40}$ & $-6.26 \times 10^{4}$ & $-3.05 \times 10^{3}$ & $-2.28 \times 10^{3}$ & $-3.60 \times 10^{4}$ \\
$\delta \dot{\omega}_{\mathrm{N} 40}$ & $4.55 \times 10^{-1}$ & $2.22 \times 10^{-2}$ & $1.66 \times 10^{-2}$ & $2.62 \times 10^{-1}$ \\
\hline \multicolumn{5}{c}{${ }^{*} \alpha=10^{-10}$} \\
\end{tabular}


The three accelerometer elements, based on the ISA (Italian Spring Accelerometer) model developed by the IAPS Experimental Gravitation Group for the BepiColombo mission [25,26], will be placed close to and aligned with the spin axis of the satellite, within the construction precision. This configuration will minimize the contribution of spurious signals due to gravitational gradients and inertial forces (see again Table 1) that are considered in the dynamics analysis shown later on. The mission design goal is to have no moving parts, thereby providing an almost constant position of the spacecraft center of mass (CM), every shift of a mass with respect to it being a source of spurious signals. Moreover, this provision will minimize (if not nullify) micro-vibrations that would excite the accelerometer sensing elements near their resonance frequency. The spacecraft should also carry all of the necessary subsystems for the satellite functions, inclusive of the attitude determination and control, through magneto torquers, of the spin axis orientation. Spin could be initiated by the release mechanism at ejection from the rocket stage and later fine-tuned with the magneto torquers.

Telecommunication with the ground will be guaranteed via an S- or X-band link, which would provide also basic tracking capabilities. Enhanced tracking will be obtained by equipping the spacecraft with a global navigation satellite system (GNSS) receiver [33]. High-precision spacecraft orbit determination will be made possible by equipping it with a set of retroreflectors for laser ranging from the International Laser Ranging Service (ILRS) stations [34].

The baseline for the mission is a minimal duration of 2-3 years, with an extended mission phase possibly aiming for a full solar cycle of 11 years. The ground segment requirements would be minimal: ground station passes for telemetry/telecommand could be limited to a few per week. The telemetry would consist mainly of GNSS, accelerometer and attitude information data, as well as housekeeping data.

In Table 3, the main features of the proposed mission, both in terms of chosen orbit and spacecraft characteristics, are shown. The spacecraft model considered here (upper part of the table) is a very schematic one and subject to be changed in further evolution of this concept.

Table 3. Main features of the proposed configuration for METRIC.

\begin{tabular}{ccc}
\cline { 2 - 3 } & Feature & Value/Type \\
\cline { 2 - 3 } & Spacecraft mass & $100 \mathrm{~kg}$ \\
Frontal area & $1 \mathrm{~m}^{2}$ \\
Solar array area & $2 \mathrm{~m}^{2}$ \\
\cline { 2 - 3 } Semimajor axis & $7.2 \times 10^{6} \mathrm{~m}$ \\
Eccentricity & 0.052 \\
Inclination * & $98.662^{\circ}$ \\
\hline Tracking & GNSS, SLR \\
Accelerations & ISA \\
\cline { 2 - 2 } * The Sun-synchronous orbit analyzed here is one of the possible high-inclination options.
\end{tabular}

\section{Expected Dynamical Environment}

As any Earth-orbiting spacecraft, METRIC would be subject to a complex dynamical environment, with gravitational and non-gravitational sources affecting its orbital motion. These sources have to be properly accounted for, in order to assess in a realistic way what signals are reasonably measurable and to design a measurement procedure (e.g., POD) able to extract these (small) signals. In this section, we are going to broadly describe these models, with a particular emphasis on what kind of signals can be extracted with the proposed mission design. The main quantitative estimates are summarized in Tables 1 and 2, to which we point the reader for a quick assessment of the main physical quantities at play. 


\subsection{Newtonian Gravitation}

Orbiting at a relatively low altitude, METRIC will be sensitive to a high degree to the Earth's mass distribution inhomogeneities. These are customarily expressed via a spherical harmonics expansion of the gravitational potential, and several models are available that describe this potential, providing the so-called Stokes coefficients $\bar{C}_{l m}$ and $\bar{S}_{l m}$ up to an $l_{\max }$ (we use here the normalized coefficients $\bar{C}_{l m}$ instead of the non-normalized $C_{l m}$; the relationship with the $J_{l}$ coefficients-used for bodies with rotational symmetry and, therefore, with $m=0$-is $\left.J_{l}=-C_{l 0}=-\sqrt{2 l+1} \bar{C}_{20}\right)$. By far the most important deviation from spherical symmetry is represented by the quadrupolar $(l=2, m=0)$ part.

Due to the gravitational quadrupole, an orbiting body is subjected to 'classical' precessions of the longitude of ascending node and the argument of pericenter given at first order by $[35,36]$ :

$$
\begin{gathered}
\dot{\Omega}_{\mathrm{N} 20}=\frac{3 \sqrt{5}}{2} n\left(\frac{R_{\oplus}}{a}\right)^{2} \frac{\cos I}{\left(1-e^{2}\right)^{2}} \bar{C}_{20}, \\
\dot{\omega}_{\mathrm{N} 20}=-\frac{3 \sqrt{5}}{2} n\left(\frac{R_{\oplus}}{a}\right)^{2} \frac{1}{\left(1-e^{2}\right)^{2}}\left(\cos ^{2} I-\frac{3}{2} \sin ^{2} I+1\right) \bar{C}_{20},
\end{gathered}
$$

with $a, e, I$ and $n$ the orbit semimajor axis, eccentricity, inclination and mean motion, respectively, and $R_{\oplus}$ the Earth mean equatorial radius. Taking into account the third Kepler's law, we notice in particular their dependence on the semimajor axis: $\dot{\Omega}_{\mathrm{N} 20}, \dot{\omega}_{\mathrm{N} 20} \sim a^{-7 / 2}$. Similar equations hold for $\dot{\Omega}_{\mathrm{N} l 0}$ and $\dot{\omega}_{\mathrm{N} l 0}, l=3,4, \ldots$

Equations (2) and (3) yield the effect of the main non-spherical part of Earth's gravitational field on an orbiting body, as well as the estimation of the associated error, given the (formal or calibrated) uncertainty declared in the model itself. Under the assumption that the error $\delta \bar{C}_{20}$ dominates over the various error sources in the formulas, one can simply replace $\bar{C}_{20}$ with this quantity, to obtain the error on the precession rate values. The results are shown in the lower part of Table 2, for METRIC and three important geodetic satellites, namely LAGEOS, LAGEOS II and LARES. This comparison is useful since these three satellites are well-tracked objects around Earth and provide a useful yardstick. We adopted values for $\bar{C}_{20}, \delta \bar{C}_{20}$ from the EIGEN-GRACE02S model, obtained from data by the GRACE mission [37]. Unlike many other comparable models, EIGEN-GRACE02S has the advantage of providing calibrated (and not simply formal) uncertainties, which are expected to be closer to the 'true' uncertainties. The table also shows the corresponding values for the octupole $(l=4)$.

A precise modeling of the METRIC orbital dynamics should include also a detailed description of the time-dependent part of the geopotential, in particular the tides. It is expected that the $S_{2}$ tide (see, e.g., [38-40]) will be particularly important for the proposed orbit, possibly causing secular signals in some of the orbital elements' residual time series. While a detailed analysis of this and other tidal contributions is outside the scope of the present work, we can make the following observation. While it is true that an error in the knowledge of $S_{2}$ tidal amplitude would possibly cause an effect masking a given relativistic signal (see below), the related tidal coefficient could be also estimated in the POD. Indeed, an improvement in the knowledge of tides is among the scientific objectives of METRIC. The price to pay will be a certain degree of correlation between such an estimated parameter and other parameters of interest. This issue will be an important task of any future error analysis.

\subsection{Relativistic Gravitation}

The relativistic dynamical model shown in Equation (1) features three corrections to the gravitational dynamics of a near-Earth object, respectively the gravitoelectric, gravitomagnetic and geodetic ones. Their main contributions to the orbital dynamics consist of a secular precessional 
behavior of some of the Keplerian elements. Specifically, the gravitoelectric part acts on the pericenter according to (Schwarzschild precession [41]):

$$
\dot{\omega}_{\mathrm{Schw}}=\frac{2+2 \gamma-\beta}{3} \frac{3\left(G M_{\oplus}\right)^{3 / 2}}{c^{2} a^{5 / 2}\left(1-e^{2}\right)} .
$$

The gravitomagnetic part (Lense-Thirring precession $[42,43]$ ) acts on the node:

$$
\dot{\Omega}_{\mathrm{LT}}=\frac{1+\gamma}{2} \frac{2 G J_{\oplus}}{c^{2} a^{3}\left(1-e^{2}\right)^{3 / 2}}
$$

and again on the pericenter:

$$
\dot{\omega}_{\mathrm{LT}}=-\frac{1+\gamma}{2} \frac{6 G J_{\oplus}}{c^{2} a^{3}\left(1-e^{2}\right)^{3 / 2}} \cos I .
$$

Finally, the geodetic part involves a precession of the node $[10,44,45]$ :

$$
\dot{\Omega}_{\mathrm{dS}}=\left(\frac{1}{2}+\gamma\right) \frac{G M_{\odot}}{c^{2} R_{\oplus \odot}^{3}}\left|\left(\mathbf{V}_{\oplus}-\mathbf{V}_{\odot}\right) \times \mathbf{R}_{\oplus \odot}\right| \cos \epsilon_{\oplus} .
$$

In the previous expressions, $a, e$ and $I$ retain their previous meaning, $J_{\oplus}=\left|\mathbf{J}_{\oplus}\right|, \mathbf{R}_{\oplus \odot}$ is the Earth-Sun vector and $\mathbf{V}_{\oplus}, \mathbf{V}_{\odot}$ the Earth and Sun barycentric velocities; $\epsilon_{\oplus}$ is the obliquity of the ecliptic (about $23.44^{\circ}$ ). These expressions are valid in the wider PPN framework. The parameters $\beta$ and $\gamma$ are considered to be the most important among the PPN ones; they amount exactly to unity in general relativity. Experiments place rather strict bounds on them: $\beta-1 \sim 10^{-5}$ and $\gamma-1 \sim 10^{-4}$, respectively [6] (instead of multiplying the gravitomagnetic precessions by the factor $(1+\gamma) / 2$, some authors, e.g., [10], prefer to multiply it by a generic parameter $\mu$-not one of the ten PPN parameters - in order to underline the fact that frame-dragging appears in a wider context than post-Newtonian approximation). Notice that the geodetic precession is the same for every Earth-bound satellite, depending essentially on the orbit of the Earth around the Sun.

A further dynamical effect worth mentioning, a possible Yukawa-like interaction, is not present in standard general relativity, but stems from a typical weak-field consequence of theoretical scenarios, which involve couplings of matter to fields other than the metric one. Recurrent theoretical motivations pointed to it; for a good review, see [46] and the more recent [47]. This interaction is described by a potential of the form:

$$
V_{Y}(r)=-\alpha \frac{G m_{i} m_{j}}{r} \exp \left(-\frac{r}{\lambda}\right)
$$

where $m_{i}, m_{j}$ are the masses of the two interacting bodies, $\lambda$ the typical interaction range (with a finite value; it can be considered as a Compton wavelength related to a force carrier of mass $m=\hbar / \lambda c$ ) and $\alpha$ a dimensionless parameter indicating the relative intensity of this interaction (its precise expression depends on the hypothesized interaction and could involve selected quantum numbers of the body constituents, implying possibly a violation of the equivalence principle). It can be shown that the presence of this further interaction would cause a supplementary precession of the argument of pericenter given at first order by:

$$
\dot{\omega}_{\mathrm{Y}}=\alpha \frac{n a \sqrt{1-e^{2}}}{e \lambda} \exp \left(-\frac{a}{\lambda}\right) I_{1}\left(\frac{a e}{\lambda}\right),
$$

with $I_{1}$ the modified Bessel function of the first kind (order one); see $[27,28,48]$.

Notice that the numerical value of $\dot{\omega}_{Y}$ depends on the intensity $\alpha$ of the interaction; the values shown in Table 2 were calculated conjecturing $\alpha=10^{-10}$, that is compatible with the error of the current best estimates for $\lambda$ comparable with the semimajor axis of LEO orbits [29,30]. Moreover, 
one can recast Equation (9) as a function $\alpha=\alpha\left(\lambda ; \dot{\omega}_{Y}, a, e, n\right)$, in which a hypothetical signal $\dot{\omega}_{Y}^{*}$ attributable to the Yukawa interaction would fix its coupling at a given $\lambda$. Consequently, the absence of such a signal would set a constraint for the interaction amplitude as a function of $\lambda$. Figure 2 shows such a hypothetical constraint in the case of a signal on the METRIC pericenter $\dot{\omega}_{\mathrm{Y}}^{*}$ equal to $10 \%$ of $\dot{\omega}_{\mathrm{LT}}$, i.e., equal to $6.93 \times 10^{-3}$ arcsec $y^{-1}$. The grey area, labeled as "Excluded region" in the plot, is the set of $(\lambda, \alpha)$ pairs for which we can reasonably exclude the existence of a Yukawa-like interaction in the given experiment. We may notice that a firmer constraint could only come from a detailed error budget analysis, analogously to what has been done in [30].

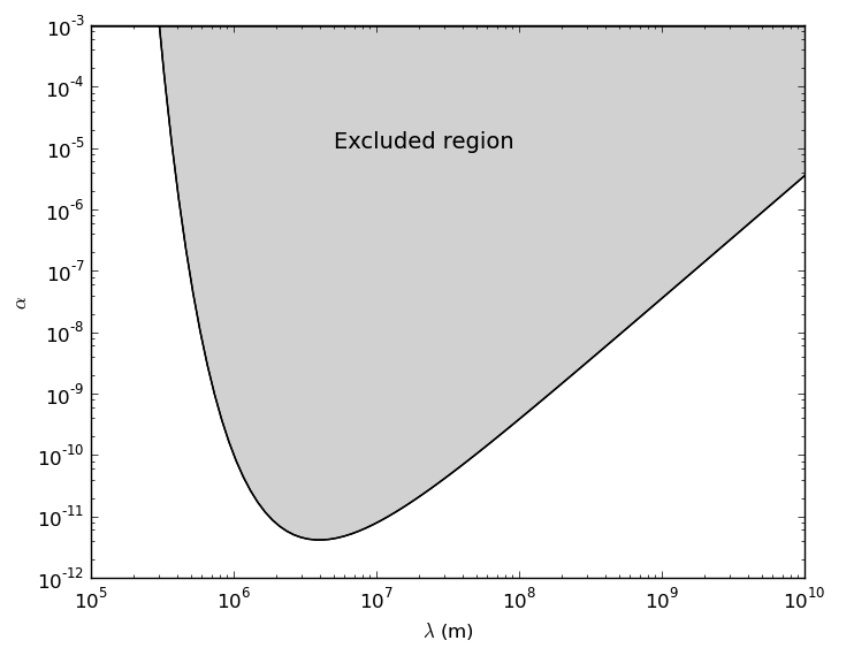

Figure 2. Hypothetical constraint on a Yukawa interaction set by a secular signal on the METRIC pericenter of $6.93 \times 10^{-3}$ arcsec $y^{-1}$. The upper, grey area would be excluded by this constraint.

Some considerations on the numerical estimates for the various effects (shown in the upper part of Table 2) are in order. The Schwarzschild precession $\dot{\omega}_{\text {Schw }}$ is of course the dominant expected relativistic signal, with the other ones at least three orders of magnitude lower. A comparison with the other satellites shows the advantage of having a lower and more eccentric orbit. This comes at the price of an increase of other effects, e.g., drag, to be modeled/measured. With these numbers, $\dot{\Omega}_{\text {LT }}$ should emerge with a signal-to-noise ratio close to unity, thereby allowing a single-satellite measurement of the gravitomagnetic field. However, this initial guess should be confirmed by a deeper analysis, e.g., considering the role of higher multipoles' uncertainties. The Yukawa signal, if any, would add power to the pericenter signal.

\subsection{Non-Gravitational Forces}

Among the non-gravitational forces currently modeled, the direct solar radiation pressure is certainly the most relevant, at least for sufficiently high orbits. This interaction (due to the reflection-absorption-diffusion of solar photons by the various spacecraft surfaces) is in general not easy to model. A well-established model for the orbital arrangement and satellite geometry it is here considered, with the solar panel hit by the direct sun illumination, while the cylinder/satellite is hit by the infrared and albedo radiation from Earth. The following formula has been used for the analysis [49]:

$$
\mathbf{a}_{\mathrm{srp}}=-\frac{\Phi_{\odot}}{c} \cos \theta \frac{A}{m}\left[(1-\epsilon) \mathbf{e}_{\odot}+2 \epsilon \cos \theta \mathbf{n}\right],
$$

with $A$ and $m$ the satellite cross-sectional illuminated area and mass, $\Phi_{\odot}$ the solar irradiance at 1 AU, $\mathbf{n}$ the unit vector normal to this area, $\mathbf{e}_{\odot}$ the unit vector pointing toward the Sun, $\theta=\angle\left(\mathbf{n}, \mathbf{e}_{\odot}\right)$ and $\epsilon$ the surface reflectivity. This model has been specialized to take into account direct illumination acting on the solar panel, infrared and albedo radiation pressure acting on the cylindrical surface of the spacecraft. 
The radiating power is calculated respectively as $\Phi_{\text {dir }}=1370 \mathrm{~W} \cdot \mathrm{m}^{-2}, \Phi_{\mathrm{alb}}=475\left(R_{\oplus} / r_{\mathrm{orb}}\right)^{2} \mathrm{~W} \cdot \mathrm{m}^{-2}$ and $\Phi_{\mathrm{IR}}=230\left(R_{\oplus} / r_{\text {orb }}\right)^{2} \mathrm{~W} \cdot \mathrm{m}^{-2}$ for direct illumination, Earth's albedo and IR radiation, respectively, $r_{\text {orb }}$ being the orbit geocentric radius.

For a spacecraft of comparatively low altitude as METRIC, the other important non-gravitational effect is due to (neutral) atmospheric drag. This is given by [49]:

$$
\mathbf{a}_{\mathrm{drag}}=-\frac{1}{2} C_{\mathrm{D}} \frac{A}{m} \rho v \mathbf{v},
$$

where apart from the previously-defined quantities, $\rho_{\mathrm{D}}$ is the atmospheric density at the satellite position, $\mathbf{v}$ is the satellite velocity with respect to the rotating atmosphere ( $v$ being its magnitude) and $C_{D}$ is the drag coefficient for a cylindrical body in free-molecular flow (it can be noticed that $C_{D}$ is not independent of the environment in which the satellite moves; what counts here is in fact the product $\left.C_{D} \rho\right)$.

\subsection{Non-Gravitational Signal Extraction}

In this section, we present a dynamical analysis to show that the drag acceleration can be extracted from the total acceleration measured by the accelerometer package, which includes the contributions associated with the rotational dynamics of the spacecraft, the imperfect centering of the three accelerometer elements, the gravity gradients and the solar radiation pressure. A model has been constructed to describe the rotational dynamics of the satellite and the effects of air drag, solar radiation pressure, gravity gradients and inertial forces. The actual geometry of the proposed satellite, consisting of a circular solar panel facing the sun rays and a cylindrical satellite body in the shadow of the solar panel (see Figure 1), was considered in modeling the effects of the solar radiation pressure and the atmospheric drag acting on the spacecraft. Moreover, the model takes into account the imperfections in the alignment of the $\mathrm{z}$-body axis (along which the accelerometers are ideally placed) with respect to the angular momentum of the satellite and realistic offset positions and centering errors of the sensing masses of the accelerometers with respect to the CM of the satellite and the z-axis. The goal of the model is to see whether the in-plane component of the drag acceleration can be extracted from the output of the $x$-body and $y$-body accelerometer sensing elements and resolved out of the total acceleration measured by the accelerometers. Moreover, the inertia characteristics of the spacecraft are important in limiting and separating in frequency the effects of the rotational dynamics in the presence of realistic centering errors and misalignments.

In the following, we show results for an axially-symmetric spacecraft with a longitudinal (about the z-axis) moment of inertia that is $20 \%$ higher than the transverse moment of inertia. The maximum-axis-spinner will be spin stable, and the free-precession frequency will be lower by a factor of five (thanks to the inertia ratio selected) than the spin frequency, thus confining the rotational-dynamics effects to lower frequencies. The above ratio of moments of inertia is not a mandatory value, but it is a valid option. We assume that the $z$-axis accelerometer element is the closest to the CM, while the $\mathrm{x}$-axis and $\mathrm{y}$-axis sensing masses are necessarily further away from the CM (see later on the description of the accelerometer). Moreover, we assume a spin period of $120 \mathrm{~s}$ (i.e., a frequency of $8.3 \times 10^{-3} \mathrm{~Hz}$ ) for the satellite and conservative values for the position imperfections and misalignment of the accelerometer sensing masses as follows: $1 \mathrm{~mm}$ off the spin axis along the $x$ and $y$ axes, $5 \mathrm{~cm}$ from the CM along the $\mathrm{z}$-axis for the sensing masses furthest from the CM and angular misalignments of the sensing axes of $1 \times 10^{-4} \mathrm{rad}$.

The air density model adopted for the simulations shown here is MSIS-86 [50]. Figure 3 shows the altitude profile and the air density predicted by the model for average solar activity, the drag-related accelerations in the local vertical-local horizontal (LV-LH) frame and the orbital velocity, all vs time. Air drag forces will change in absolute terms by using a different density model and, more importantly, a different value of solar activity, yet without changing the nature of the problem. The goal of these computations is to demonstrate that the air drag force can be resolved 
out of the total acceleration measured by the accelerometers that includes comparatively stronger contributions from the other noise sources. The accelerations measured by the onboard accelerometers, shown in Figure 4, are the sum of air drag, solar radiation, gravity gradient and spin dynamics contributions associated with sensing masses of the accelerometers that are neither aligned, nor coincident with the spin axis. The (simulated) air drag acceleration components are also shown. The total accelerations measured by the $\mathrm{x}$-body and y-body accelerometer elements (top panel of Figure 4) are first de-biased and then referred to the LV-LH frame (this frame is also known as the Gauss frame) that follows the CM of the satellite (see Figure 5), by using the phase information that in practice will be provided by the on-board attitude measurement system. A moving average filter was then utilized to extract the local horizontal (LH) drag component. This component, almost aligned with the flight direction (FD), is by far the most important drag component acting on the satellite when compared to the much smaller components, at right angles to the FD, associated with the rotating atmosphere and the orbital eccentricity. The bottom panel of Figure 5 depicts the total acceleration along LH, the simulated LH drag acceleration component (labeled LH drag sim) and the extracted/resolved LH drag acceleration (labeled LH drag res) over one orbit. The resolved acceleration component follows closely the simulated profile of the LH air drag acceleration once the filter converges in about one spin period (with the filter time lag accounted for), demonstrating the validity of the procedure adopted for extracting the drag-related acceleration "signal". Table 1 shows the range of acceleration magnitudes acting on the system grouped according to their type. It can be noted that some components, like the solar-radiation and the spin-related accelerations, are much stronger than the smallest expected drag acceleration. However, thanks to the $90^{\circ}$ phase shift and spin modulation for the former acceleration and the frequency separation for the latter, the LH drag component can be extracted out of the higher "noise" components.
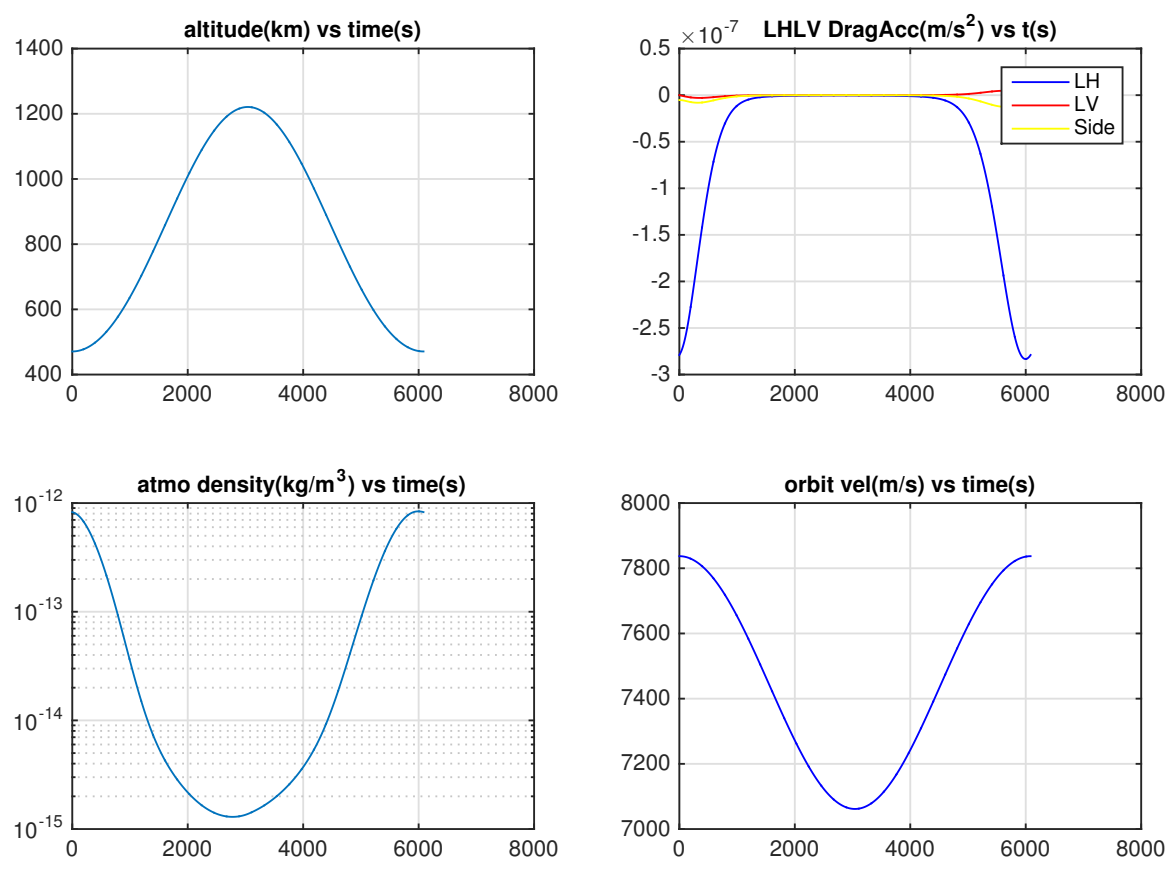

Figure 3. Altitude profile (upper left); atmospheric density (lower left); drag acceleration components (upper right); and orbital velocity (lower right). All quantities are plotted over one orbit. 

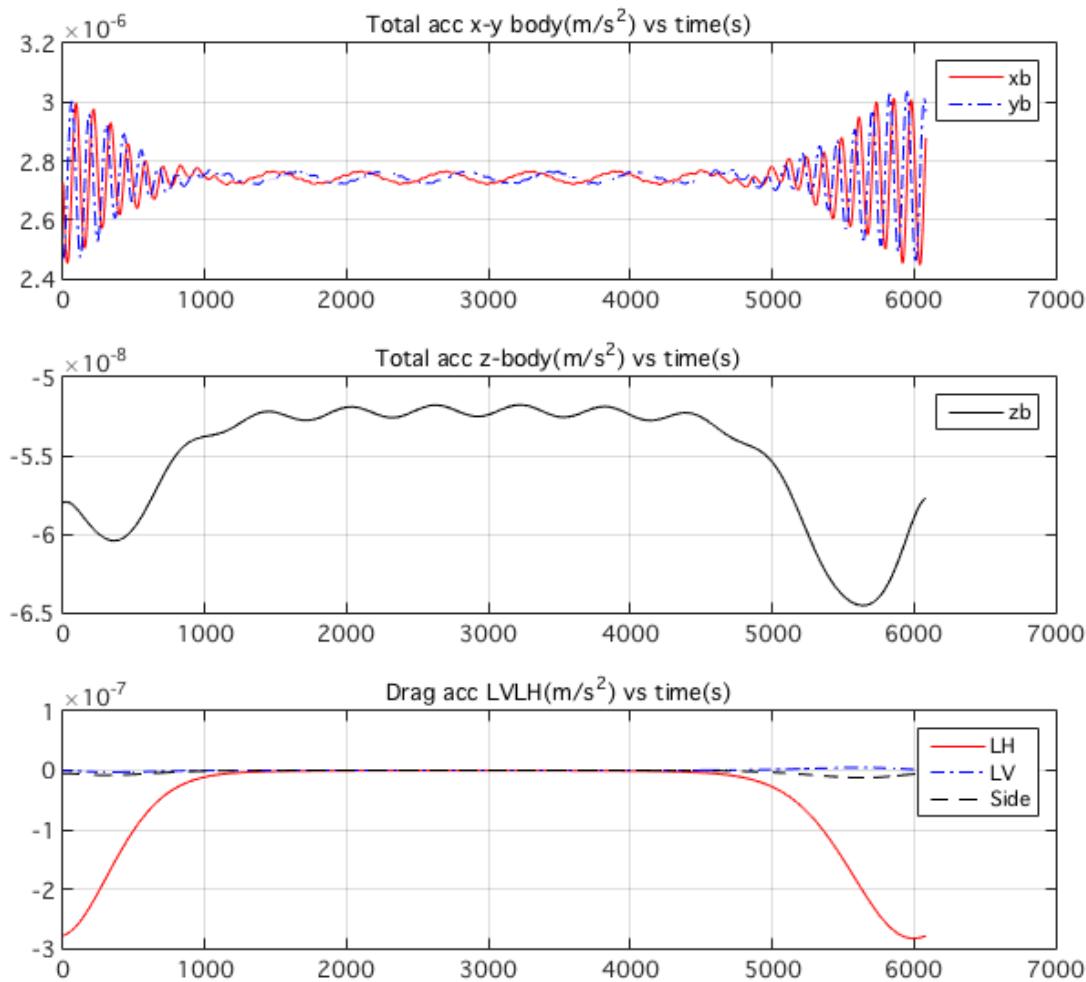

Figure 4. Total acceleration measured by $x$-body and y-body (top) and z-body (middle) accelerometer sensing elements and simulated air drag acceleration components (bottom), over one orbit.
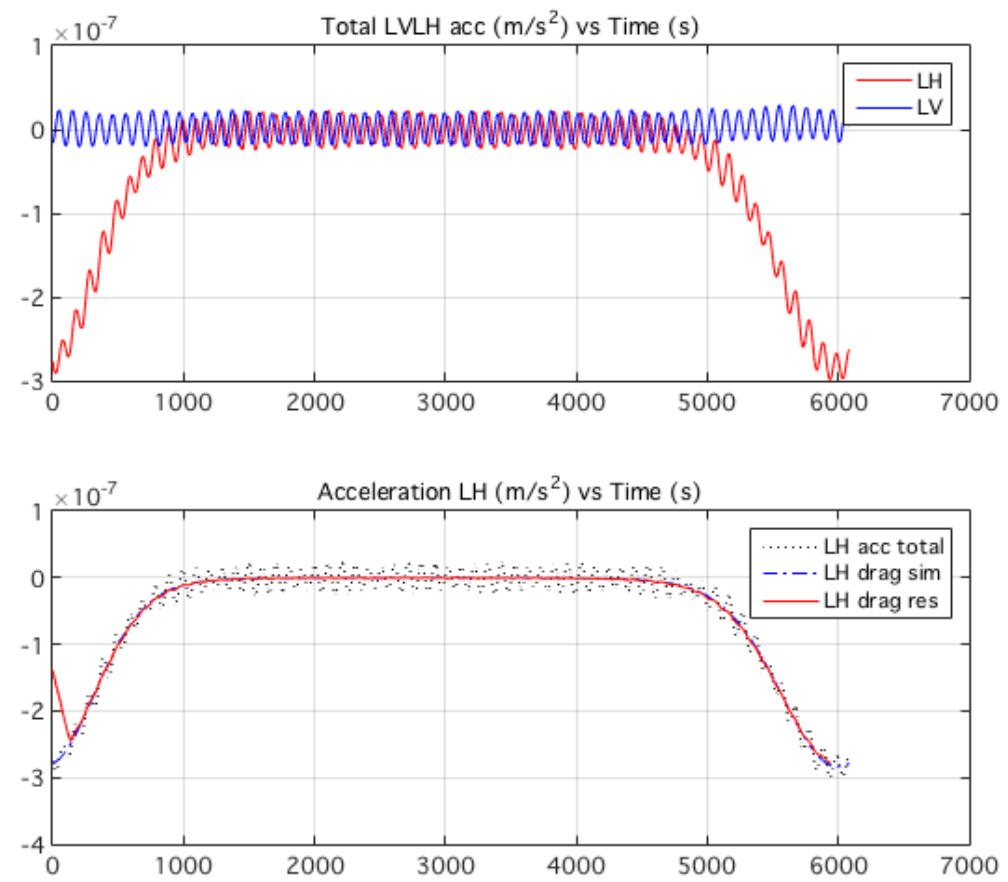

Figure 5. (Top) Total acceleration components in the LV-LH frame; (bottom) total acceleration along LH, simulated LH drag acceleration component (labeled "LH drag sim") and resolved LH drag acceleration (labeled "LH drag res") over one orbit. 


\section{The Accelerometer Package}

In order to fine-tune the characteristics of the accelerometer model for METRIC, it is important to estimate the spectral features of the expected signals. A requirements envelope comes from Table 1: the expected LH component lies in the amplitude range $3 \times 10^{-10}$ to $3 \times 10^{-7} \mathrm{~m} \cdot \mathrm{s}^{-2}$. A maximum sampling frequency of $0.1 \mathrm{~Hz}$ corresponds to density variations in the atmosphere with a horizontal scale of roughly $80 \mathrm{~km}$, which would be a significant improvement with respect to the present knowledge. These figures (which could be fine-tuned by a more detailed analysis outside the scope of this work) fit well into what is currently reachable with state-of-the-art acceleration measurement devices. In particular, they could be attainable with an adaptation of the ISA BepiColombo accelerometer to the proposed mission. Very important in this respect is the fact that METRIC would offer a stable environment for the instruments it will host; indeed, a relevant part of the accelerometer error budget in that case is given by spurious signals coming from the on-board environment itself (see, e.g., [26,51]).

The three-axis, high-sensitivity accelerometer to be hosted inside the METRIC spacecraft is ISA, a three-axis, high-sensitivity device, developed for space applications, but suited also for ground (e.g., geophysical) uses. The instrument consists of three one-dimensional sensing elements, one for each spatial direction, arranged such that the sensing directions are orthogonal to one another (see Figure 6). Each sensing element is basically a one-dimensional harmonic oscillator, in which a sensing mass is connected to an external frame with a flexural spring; the entire oscillator is obtained by the manufacturing of a single piece of aluminum. The central mass is in between two pick-up plates to form a two-face capacitor (see Figure 7). The two-face capacitor and the other two fixed ones are part of a capacitive bridge for signal read-out. An acceleration acting on the system (its component along the normal to the plates) induces a motion of the central mass and unbalances the bridge. The corresponding signal is then read out and properly amplified and filtered. The signals coming from each accelerometer element can therefore be combined to obtain the acceleration vector at a reference point of the accelerometer. This arrangement implies taking into account the effects of inertial accelerations and gravity gradients, as was done in the model employed in Section 4.4.

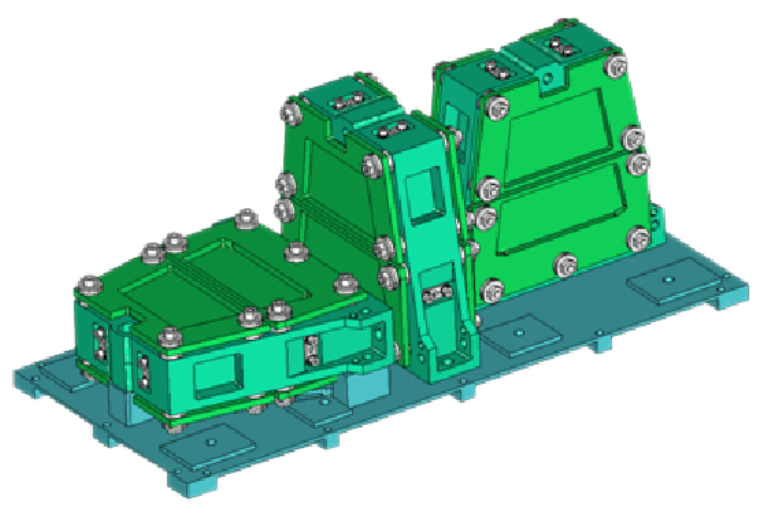

Figure 6. Rendering of the three ISA sensing elements arranged in a typical flight configuration. The acceleration vector on a reference point is reconstructed from the three measured components. Image courtesy of ISA BepiColombo Science Team.

The design sensitivity of the ISA accelerometer developed for BepiColombo (this is a good starting point figure: it has been specifically designed for space use) is of the order of $1 \times 10^{-9} \mathrm{~m} \cdot \mathrm{s}^{-2} \cdot \mathrm{Hz}^{-1 / 2}$; this level has been verified on the ground over many tests with accelerometer prototypes assembled in a differential configuration [26], in a wide band of frequencies below $1 \times 10^{-1} \mathrm{~Hz}$. Along with the pick-up plates, each sensing element has another two plates able to give the mass a known acceleration signal, thereby providing an internal calibration means (see again Figure 7). This 
calibration can be complemented with an a posteriori bias and (possibly) drift adjustment via the POD (see, e.g., [52]). In order to reduce as much as possible the inertial signals, the accelerometer should be positioned as close as possible to the satellite spin axis, as well as in a low-noise mechanical and thermal environment (both mechanical and thermal environment are expected to be reasonably quiet, due respectively to the minimization of moving parts onboard the spacecraft and its nearly constant attitude with respect to the Sun).

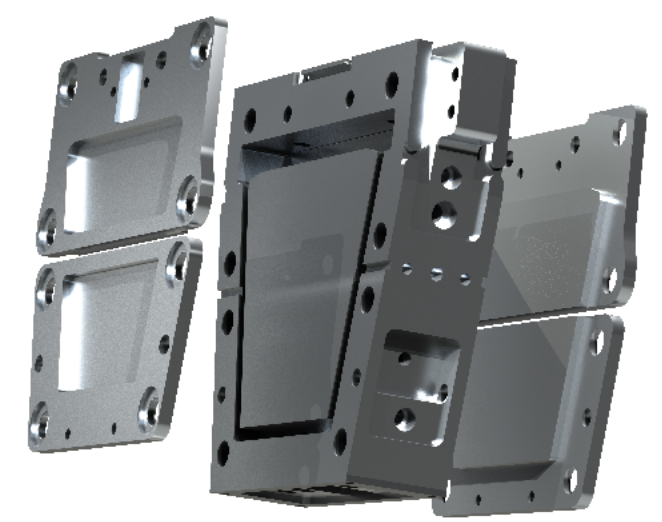

Figure 7. Rendering of a single ISA sensing element. At the center of the assembly is the sensing mass connected to the external frame and surrounded by two couples of read-out and control plates. Image courtesy of ISA BepiColombo Science Team.

\section{Tracking Options}

METRIC requires the best tracking techniques available for near-Earth satellites; natural choices would be therefore GNSS and SLR. It has to be noticed that these are two fundamental space geodesy techniques, routinely used for the realization and maintenance of the ITRF [20]. Their inclusion is therefore a minimum requirement in order to satisfy the fourth scientific objective on the tie among different space geodesy techniques. We briefly discuss here their main capabilities and limitations; these are also summarized in Table 4.

Table 4. Main features of the proposed tracking techniques.

\begin{tabular}{|c|c|}
\hline Technique & Features \\
\hline GNSS & $\begin{array}{l}\text { Rather simple and inexpensive } \\
\text { Coverage: almost continuous } \\
\text { Observable: pseudo-range (code and phase), navigation solution with sub-cm precision } \\
\text { POD: sub-dm }\end{array}$ \\
\hline SLR & $\begin{array}{l}\text { Very simple in principle, but requires ILRS dedicated tracking } \\
\text { Coverage: depends on observation geometry, atmospheric conditions and station schedule } \\
\text { Observable: range, } \sim 1 \mathrm{~mm} \text { precision } \\
\text { POD: sub-dm, approaching the cm level with best models }\end{array}$ \\
\hline
\end{tabular}

A GNSS receiver on board would provide in a rather simple way a kinematic orbit for the spacecraft, almost continuous in time. By "kinematic" we mean the orbit is obtained by the navigation solution, and not by an integration of the satellite equation of motion and a fit to the tracking data, as in the dynamic one. Experience with the CHAMP mission shows that with a navigation solution of sub-cm precision, an orbit solution with a sub-dm level can be achieved, using GPS code and phase data [53,54]. GNSS tracking has indeed the advantage of providing a uniform geometric solution of good quality; this would therefore constitute an adequately accurate basis to be used as a starting point for further analyses. 
The SLR technique (see Figure 8) employs the timing of the round-trip travel of laser pulses from ground stations (ILRS network) to the satellite (equipped with retroreflectors) and back; the basic observable is therefore a range (instantaneous station-satellite distance). Usually the so-called normal points are used, that are basically an average of laser ranges over a definite time interval (bin); they are characterized by the fact that their random error is reduced to that of the mean of the bin. The precision of a single normal point is outstanding: usually, it is below the $\mathrm{cm}$ and, nowadays, approaches the $\mathrm{mm}$ level. The resulting POD is model-limited, and could approach the $\mathrm{cm}$. The coverage of SLR observations is however non-uniform, being dependent on observation geometry, atmospheric conditions and station schedule.

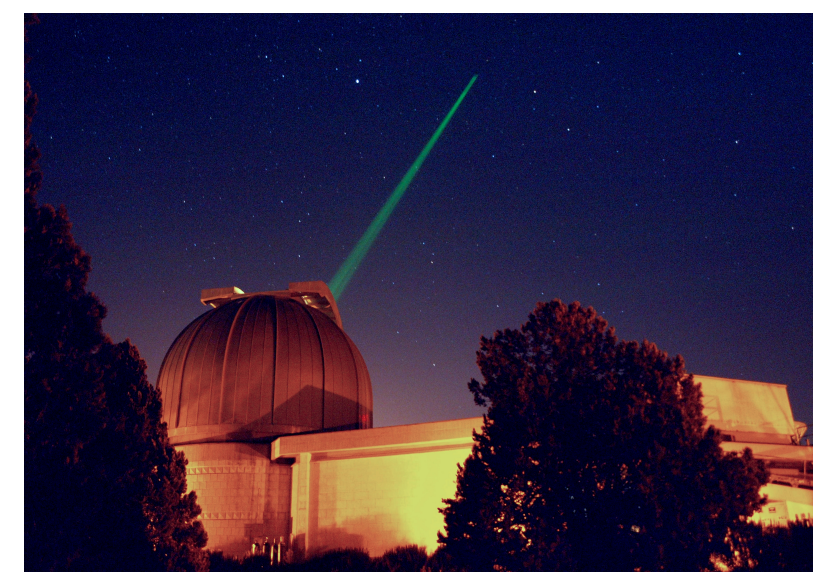

Figure 8. Matera laser station during a pass. Photo by Franco Ambrico. Courtesy Giuseppe Bianco, ASI-CGS.

These two techniques are therefore in a way complementary to each other: the POD could include both to employ their best observations and models. To this minimal set, other instrumentation, related to the other two space geodesy techniques-namely very long baseline interferometry (VLBI) and Doppler orbitography and radio-positioning integrated by satellite (DORIS)—could in principle be added at the price of increasing the complexity and cost of the mission. Consequently, in this case, the spacecraft would become a full-fledged in-orbit geodetic station.

\section{Data Analysis Issues}

The data analysis procedures to be implemented should be functional for the scientific objectives of the mission as discussed in Section 2. All of these would require POD; the third and fourth objectives would require further analysis activities. We briefly discuss here their main peculiarities.

\subsection{Precise Orbit Determination}

The POD based on the various tracking data types would be the basis of any subsequent analysis. As customary for geodetic missions, a multi-arc technique [55] could be employed, in which for each arc, a dynamical model is fitted to the data, obtaining the precise spacecraft ephemerides and estimates of selected parameters. A number of software packages has been developed, which are able to perform a POD by processing at the same time GNSS and SLR data; among them, we notice Geodyn II (NASA/GSFC) [56,57]. Said in other words, what is required is a least-squares solution of:

$$
O_{i}-M_{i}=-\sum_{j} \frac{\partial M_{i}}{\partial P_{j}} d P_{j}+d O_{i}
$$

where $O_{i}$ and $M_{i}$ are, respectively, the observations (e.g., pseudo-ranges or ranges) and their corresponding modeled values, $d P_{j}$ the corrections to the vector $\mathbf{P}$ of parameters to be estimated 
and $d O_{i}$ are the errors associated with each observation. The $d O_{i}$ account for both the noise in the observations and the incompleteness of the dynamical model. A well-conducted POD minimizes the residuals $O_{i}-M_{i}$, providing at the same time unbiased (for what is possible) estimates of the selected parameters. Relevant scientific information could be obtained by the time series of the estimated parameters or by an a posteriori residuals analysis (following, e.g., the method outlined in [58]).

The information content of the tracking data is related to [49]:

- $\quad$ spacecraft dynamics;

- measurement procedures;

- reference frames.

Each of these should be properly accounted for in the POD procedure, in order to extract as best as possible the information contained in the data. The non-gravitational part of the modelization set will be provided directly by the acceleration measurements. The proper use of the acceleration data would depend on a dedicated data reduction procedure, including the projection of the acceleration components to selected directions required by the POD software, which requires the attitude information.

\subsection{Drag Measurements}

The estimation of the atmospheric density from the drag-related acceleration data is obviously dependent on the knowledge of the satellite cross-section in the flight direction and the aerodynamic drag coefficient; the parameter provided by the accelerometer data is indeed $C_{D} \rho$. Because METRIC is a stabilized satellite spinning along the axis orthogonal to the orbital plane and with cylindrical symmetry, the cross-section is well defined. The estimate of the drag coefficient in free-molecular flow is subject to the usual experimental uncertainties that are common to any satellite in orbit and dependent, for the re-emission contribution, on surface properties and temperature. However, METRIC has the advantages of having a simple, spin-symmetric geometry and will have a stable temperature (that affects the re-emission of the neutral particles and hence the drag coefficient) if a Sun-synchronous orbit is utilized. A polar orbit will be less favorable from this point of view, but it could be a better alternative for the determination of the relativistic effects.

\subsection{Multi-Space Geodesy Techniques Tie}

The establishment of an ITRF solution is a complex procedure in which individual solutions for each space geodesy technique are obtained from the respective analysis centers; these long-term single-technique solutions are subsequently combined in order to obtain the ITRF solution [20]. This combination relies also on the so-called local ties at co-location sites. These are geodetic sites where more than one space geodetic technique is present (e.g., GNSS and SLR); as the name suggests, they are fundamental in bringing together the various single-technique solutions via a set of Helmert transformations. The local ties are a critical factor in this respect, and their maintenance (e.g., in the case of the GNSS phase center eccentricities) is a continuous challenge. METRIC would indeed offer an important contribution to this problem, constituting an in-space tie potentially very well determined from the metrological point of view.

\section{Outlook}

METRIC appears as a multidisciplinary mission, able in principle to contribute to geophysics, fundamental physics, navigation and orbit evolution prediction. Several steps would be required in any case to develop the basic ideas previously outlined into a firm mission proposal. A more detailed analysis will be necessary to select an optimum orbital configuration, as well as to fine-tune the accelerometer performance requirements (in terms of both sensitivity and spectral features). A comprehensive set of simulations will be very important, whereby the main hypotheses on orbital dynamics and data analysis could be explored. Particular attention should be devoted to the sensitivity 
of relativistic signals to higher zonal harmonics and tidal lines. The simulations should provide also information on the tracking requirements necessary to fulfill the scientific objectives of the mission. The very basic concept of spacecraft as outlined above should be further developed into a consolidated instrument complement, possibly increasing the number of space geodetic instrumentation hosted on board.

\section{Conclusions}

This work outlines a mission concept, named METRIC, for a compact spacecraft dedicated to the study of a number of key topics in the fields of fundamental physics and geophysics, including the test of selected predictions of general relativity theory and direct measurements of atmospheric drag in an altitude range of interest for satellite lifetime predictions. A preliminary analysis of the expected dynamical environment shows very interesting prospects for the advancement of knowledge in these fields. A very interesting by-product of this mission would be the realization of an in-orbit metrological platform useful to establish a precise tie between different space geodesy techniques, with potentially important contributions to the Terrestrial Reference Frame solution (e.g., to navigation). The basic elements of the proposal (in terms of both orbit and spacecraft configuration) have been discussed, with an outlook towards future activities.

Acknowledgments: The authors acknowledge the ISA Science Team for specifications and renderings of the ISA accelerometer.

Author Contributions: Roberto Peron and Enrico C. Lorenzini contributed equally to this work. The mission concept was originally proposed by Enrico C. Lorenzini.

Conflicts of Interest: The authors declare no conflict of interest.

\section{Abbreviations}

The following abbreviations are used in this manuscript:

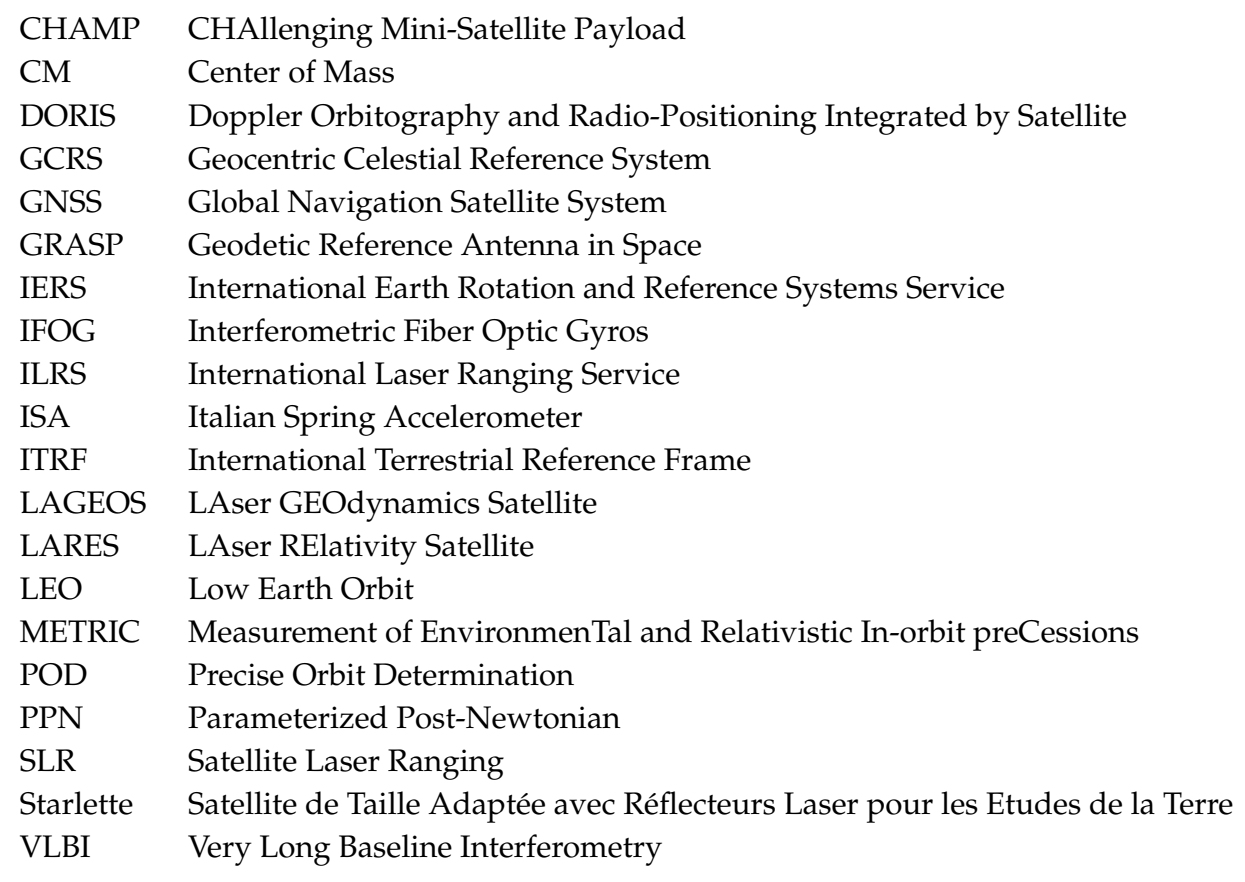

\section{References}

1. Abbott, B.P.; Abbott, R.; Abbott, T.D.; Abernathy, M.R.; Acernese, F.; Ackley, K.; Adams, C.; Adams, T.; Addesso, P.; Adhikari, R.X.; et al. Observation of Gravitational Waves from a Binary Black Hole Merger. Phys. Rev. Lett. 2016, 116, 061102. 
2. Abbott, B.P.; Abbott, R.; Abbott, T.D.; Abernathy, M.R.; Acernese, F.; Ackley, K.; Adams, C.; Adams, T.; Addesso, P.; Adhikari, R.X.; et al. GW151226: Observation of Gravitational Waves from a 22-Solar-Mass Binary Black Hole Coalescence. Phys. Rev. Lett. 2016, 116, 241103.

3. Abbott, B.P.; Abbott, R.; Abbott, T.D.; Acernese, F.; Ackley, K.; Adams, C.; Adams, T.; Addesso, P.; Adhikari, R.X.; Adya, V.B.; et al. GW170104: Observation of a 50-Solar-Mass Binary Black Hole Coalescence at Redshift 0.2. Phys. Rev. Lett. 2017, 118, 221101.

4. Abbott, B.P.; Abbott, R.; Abbott, T.D.; Abernathy, M.R.; Acernese, F.; Ackley, K.; Adams, C.; Adams, T.; Addesso, P.; Adhikari, R.X.; et al. Tests of General Relativity with GW150914. Phys. Rev. Lett. 2016, 116, 221101.

5. Turyshev, S.G. Experimental Tests of General Relativity. Annu. Rev. Nucl. Part. Sci. 2008, 58, $207-248$.

6. Will, C.M. The Confrontation between General Relativity and Experiment. Living Rev. Relativ. 2014, 17, 4.

7. Peron, R. Fundamental Physics with the LAGEOS Satellites. In Gravity: Where Do We Stand? Peron, R., Colpi, M., Gorini, V., Moschella, U., Eds.; Springer: Berlin, Germany, 2016; p. 167, ISBN 978-3-319-20223-5.

8. Poisson, E.; Will, C.M. Gravity; Cambridge University Press: Cambridge, UK, 2014.

9. Ciufolini, I.; Wheeler, J.A. Gravitation and Inertia; Princeton University Press: Princeton, NJ, USA, 1995.

10. Lucchesi, D.M.; Anselmo, L.; Bassan, M.; Pardini, C.; Peron, R.; Pucacco, G.; Visco, M. Testing the gravitational interaction in the field of the Earth via satellite laser ranging and the Laser Ranged Satellites Experiment (LARASE). Class. Quantum Gravity 2015, 32, 155012.

11. Pardini, C.; Anselmo, L.; Moe, K.; Moe, M.M. Drag and energy accommodation coefficients during sunspot maximum. Adv. Space Res. 2010, 45, 638-650.

12. Pardini, C.; Moe, K.; Anselmo, L. Thermospheric density model biases at the 23rd sunspot maximum. Planet. Space Sci. 2012, 67, 130-146.

13. Kessler, D.J. Collisional cascading: The limits of population growth in low earth orbit. Adv. Space Res. 1991, 11, 63-66.

14. Peron, R.; Iafolla, V.; Fiorenza, E.; Lefevre, C.; Lucchesi, D.; Lucente, M.; Magnafico, C.; Santoli, F.; Lorenzini, E.; Anselmo, L.; et al. Investigating fundamental physics and the space environment with a dedicated Earth-orbiting spacecraft. In Proceedings of the IEEE Metrology for Aerospace (MetroAeroSpace), Benevento, Italy, 29-30 May 2014; pp. 255-259.

15. Petit, G.; Luzum, B. IERS Conventions (2010); IERS Technical Note 36, IERS, Frankfurt am Main; Verlag des Bundesamts für Kartographie und Geodäsie: Frankfurt am Main, Germany, 2010.

16. Soffel, M.; Klioner, S.A.; Petit, G.; Wolf, P.; Kopeikin, S.M.; Bretagnon, P.; Brumberg, V.A.; Capitaine, N.; Damour, T.; Fukushima, T.; et al. The IAU 2000 Resolutions for Astrometry, Celestial Mechanics, and Metrology in the Relativistic Framework: Explanatory Supplement. Astron. J. 2003, 126, 2687-2706.

17. Soffel, M.H. Relativity in Astrometry, Celestial Mechanics and Geodesy; Springer: Berlin, Germany, 1989.

18. Cheng, M.K.; Shum, C.K.; Eanes, R.J.; Schutz, B.E.; Tapley, B.D. Long-period perturbations in Starlette orbit and tide solution. J. Geophys. Res. 1990, 95, 8723-8736.

19. Bar-Sever, Y.; Haines, B.; Bertiger, W.; Desai, S.; Wu, S. Geodetic reference antenna in space (GRASP)—A mission to enhance spac-based geodesy. In Proceedings of the COSPAR Colloquium: Scientific and Fundamental Aspects of The Galileo Program, Padua, Italy, 14-16 October 2009.

20. Altamimi, Z.; Collilieux, X.; Métivier, L. ITRF2008: An improved solution of the international terrestrial reference frame. J. Geod. 2011, 85, 457-473.

21. Cohen, S.C.; King, R.W.; Kolenkiewicz, R.; Rosen, R.D.; Schutz, B.E. LAGEOS scientific results. J. Geophys. Res. 1985, 90, 9215-9438.

22. Reigber, C.; Lühr, H.; Schwintzer, P. CHAMP mission status. Adv. Space Res. 2002, 30, 129-134.

23. Touboul, P.; Willemenot, E.; Foulon, B.; Josselin, V. Accelerometers for CHAMP, GRACE and GOCE space missions: Synergy and evolution. Boll. Geof. Teor. Appl. 1999, 40, 321-327.

24. Milani, A.; Rossi, A.; Vokrouhlický, D.; Villani, D.; Bonanno, C. Gravity field and rotation state of Mercury from the BepiColombo Radio Science Experiments. Planet. Space Sci. 2001, 49, 1579-1596.

25. Iafolla, V.; Nozzoli, S. Italian spring accelerometer (ISA) a high sensitive accelerometer for "BepiColombo" ESA CORNERSTONE. Planet. Space Sci. 2001, 49, 1609-1617.

26. Iafolla, V.; Fiorenza, E.; Lefevre, C.; Morbidini, A.; Nozzoli, S.; Peron, R.; Persichini, M.; Reale, A.; Santoli, F. Italian Spring Accelerometer (ISA): A fundamental support to BepiColombo Radio Science Experiments. Planet. Space Sci. 2010, 58, 300-308. 
27. Lucchesi, D.M. LAGEOS II perigee shift and Schwarzschild gravitoelectric field. Phys. Lett. A 2003, 318, 234-240.

28. Lucchesi, D.M. The LAGEOS satellites orbit and Yukawa-like interactions. Adv. Space Res. 2011, 47, 1232-1237.

29. Lucchesi, D.M.; Peron, R. Accurate Measurement in the Field of the Earth of the General-Relativistic Precession of the LAGEOS II Pericenter and New Constraints on Non-Newtonian Gravity. Phys. Rev. Lett. 2010, 105, 231103.

30. Lucchesi, D.M.; Peron, R. LAGEOS II pericenter general relativistic precession (1993-2005): Error budget and constraints in gravitational physics. Phys. Rev. D 2014, 89, 082002.

31. Vespe, F.; Rutigliano, P. The improvement of the Earth gravity field estimation and its benefits in the atmosphere and fundamental physics. Adv. Space Res. 2005, 36, 472-485.

32. Ciufolini, I.; Pavlis, E.C.; Peron, R. Determination of frame-dragging using Earth gravity models from CHAMP and GRACE. New Astron. 2006, 11, 527-550.

33. Dow, J.M.; Neilan, R.E.; Rizos, C. The International GNSS Service in a changing landscape of Global Navigation Satellite Systems. J. Geod. 2009, 83, 191-198.

34. Pearlman, M.R.; Degnan, J.J.; Bosworth, J.M. The International Laser Ranging Service. Adv. Space Res. 2002, 30, 135-143.

35. Kaula, W.M. Theory of Satellite Geodesy. Applications of Satellites to Geodesy; Blaisdell: Waltham, MA, USA, 1966.

36. Iorio, L. The Impact of the Static Part of the Earth's Gravity Field on Some Tests of General Relativity with Satellite Laser Ranging. Celest. Mech. Dyn. Astron. 2003, 86, 277-294.

37. Reigber, C.; Schmidt, R.; Flechtner, F.; König, R.; Meyer, U.; Neumayer, K.H.; Schwintzer, P.; Zhu, S.Y. An Earth gravity field model complete to degree and order 150 from GRACE: EIGEN-GRACE02S. J. Geodyn. 2005, 39, 1-10.

38. Agnew, D. 3.06-Earth Tides. In Treatise on Geophysics, 2nd ed.; Schubert, G., Ed.; Elsevier: Oxford, UK, 2015; pp. 151-178.

39. Felsentreger, T.L.; Marsh, J.G.; Agreen, R.W. Analyses of the solid earth and ocean tidal perturbations on the orbits of the Geos 1 and Geos 2 satellites. J. Geophys. Res. 1976, 81, 2557-2563.

40. Parke, M.E.; Stewart, R.H.; Farless, D.L.; Cartwright, D.E. On the choice of orbits for an altimetric satellite to study ocean circulation and tides. J. Geophys. Res. 1987, 92, 11.

41. Rubincam, D.P. General relativity and satellite orbits-The motion of a test particle in the Schwarzschild metric. Celest. Mech. 1977, 15, 21-33.

42. Lense, J.; Thirring, H. Über den Einfluß der Eigenrotation der Zentralkörper auf die Bewegung der Planeten und Monde nach der Einsteinschen Gravitationstheorieh. Phys. Z. 1918, 19, 156. (In Germany)

43. Mashhoon, B.; Hehl, F.W.; Theiss, D.S. On the gravitational effects of rotating masses-The Thirring-Lense Papers. Gen. Relativ. Gravit. 1984, 16, 711-750.

44. De Sitter, W. On Einstein's theory of gravitation and its astronomical consequences. Second paper. Mon. Not. R. Astron. Soc. 1916, 77, 155-184.

45. Huang, C.; Ries, J.C.; Tapley, B.D.; Watkins, M.M. Relativistic effects for near-earth satellite orbit determination. Celest. Mech. Dyn. Astron. 1990, 48, 167-185.

46. Fischbach, E.; Talmadge, C. Six years of the fifth force. Nature 1992, 356, 207-215.

47. Adelberger, E.G.; Heckel, B.R.; Nelson, A.E. Tests of the Gravitational Inverse-Square Law. Annu. Rev. Nucl. Part. Sci. 2003, 53, 77-121.

48. Deng, X.M.; Xie, Y. Yukawa effects on the clock onboard a drag-free satellite. MNRAS 2013, 431, 3236-3239, arXiv:gr-qc/1303.0949.

49. Montenbruck, O.; Gill, E. Satellite Orbits. Models, Methods and Applications; Springer: Berlin, Germany, 2000.

50. Rees, D. COSPAR International Reference Atmosphere: 1986. Part 1: Thermosphere models. Adv. Space Res. 1988, 8, 476 .

51. Iafolla, V.; Lucchesi, D.M.; Nozzoli, S.; Santoli, F. ISA accelerometer onboard the Mercury Planetary Orbiter: Error budget. Celest. Mech. Dyn. Astron. 2007, 97, 165-187.

52. Van den Ijssel, J.; Visser, P. Performance of GPS-based accelerometry: CHAMP and GRACE. Adv. Space Res. 2007, 39, 1597-1603.

53. Van den Ijssel, J.; Visser, P.; Patiño Rodriguez, E. Champ precise orbit determination using GPS data. Adv. Space Res. 2003, 31, 1889-1895. 
54. Moore, P.; Turner, J.F.; Qiang, Z. Champ orbit determination and gravity field recovery. Adv. Space Res. 2003, 31, 1897-1903.

55. Milani, A.; Gronchi, G.F. Theory of Orbit Determination; Cambridge University Press: Cambridge, UK, 2010.

56. Putney, B.; Kolenkiewicz, R.; Smith, D.; Dunn, P.; Torrence, M.H. Precision orbit determination at the NASA Goddard Space Flight Center. Adv. Space Res. 1990, 10, 197-203.

57. Pavlis, D.E.; Luo, S.; Dahiroc, P.; McCarthy, J.J.; Luthke, S.B. GEODYN II Operations Manual; NASA GSFC: Greenbelt, MD, USA, 1998.

58. Lucchesi, D.M.; Balmino, G. The LAGEOS satellites orbital residuals determination and the Lense Thirring effect measurement. Planet. Space Sci. 2006, 54, 581-593.

(C) 2017 by the authors. Licensee MDPI, Basel, Switzerland. This article is an open access article distributed under the terms and conditions of the Creative Commons Attribution (CC BY) license (http://creativecommons.org/licenses/by/4.0/). 